\title{
La vivienda construida en serie y la influencia de la normatividad para las condiciones de habitabilidad en Ciudad Juárez, Chihuahua, México
}

\author{
Housing built in series and the influence of normativity for habitability conditions in Ciudad \\ Juarez Chihuahua, Mexico \\ Leticia Peña Barrera ${ }^{a}$, Luis Herrera Terraza Barrera ${ }^{a}$ Carmen García Gómez ${ }^{b}$
}

\begin{abstract}
:
Urban growth in Ciudad Juarez Chihuahua, Mexico, from the last two decades has been particularly in subdivisions with housing built in series. The installation of the maquiladora industry and the purpose of housing the families that work in it, favors the construction of housing developments where essentially houses with minimal dimensions are built. The objective of this paper is to analyze the habitability conditions related to the dimensions, the regulations and the abandonment of the housing of the Las Haciendas subdivision in Ciudad Juarez that contribute to the reflection on the impact on the quality of the habitat of the families that inhabit them. The regulations are a determining factor for the dimensioning of the house, because the real estate companies take these restrictions to the minimum limit in construction, thus generating problems subsequent to the habitability conditions of the house and the urban context.
\end{abstract}

Keywords:

Social housing, serial construction, normativity, habitability

\section{Resumen:}

El crecimiento urbano en Ciudad Juárez Chihuahua, México, a partir de las dos últimas décadas ha sido particularmente en fraccionamientos con vivienda construida en serie. La instalación de la industria maquiladora y el propósito de albergar las familias que laboran en ésta, propicia la construcción de desarrollos habitacionales donde esencialmente se construye vivienda con dimensiones mínimas. El objetivo del presente escrito es analizar las condiciones de habitabilidad relacionadas con las dimensiones, la normatividad y el abandono de la vivienda del fraccionamiento Las Haciendas en Ciudad Juárez, que aporten a la reflexión sobre el impacto en la calidad del hábitat de las familias que las habitan. La normativa es un factor determinante para el dimensionamiento de la vivienda, porque las inmobiliarias toman estas restricciones al límite mínimo en la construcción, generando así problemáticas subsecuentes a las condiciones de habitabilidad de la vivienda y el contexto urbano.

\section{Palabras Clave:}

Vivienda social, construcción en serie, normatividad, habitabilidad

\section{Introducción}

La instalación de la industria maquiladora, a partir de los años sesentas, y el creciente establecimiento de parques industriales en diferentes áreas de la ciudad de manera policéntrica, incorporan dentro de la dinámica de crecimiento urbano, la concentración de población, atraída por su fácil empleabilidad. Siendo esta ciudad, un atractivo laboral para las personas de otras partes del país, que emigran de las zonas rurales, primero con el interés de pasar a los Estados Unidos y luego se establecieron al encontrar oportunidades de empleo en estas empresas. Por lo que se asientan en Ciudad Juárez de manera permanente, teniendo la posibilidad de acceder a una vivienda mediante el sistema del financiamiento institucional de INFONAVIT. La dispersión y crecimiento expansivo de la ciu dad está vinculada a la política habitacional, promovida por el 
modelo neoliberal, a partir del año 2000, que lleva a la ocupación de zonas alejadas para abaratar los costos de la vivienda, con la creación de zonas periurbanas que propician la segregación pasiva, según lo mencionan Ruvalcaba y Schteingart (2012). La creación de nuevos desarrollos habitacionales de vivienda en serie, principalmente de nivel socioeconómico bajo, se presenta de forma predominante en áreas periféricas al sur y sur-oriente del centro de la Ciudad, aproximadamente a más de 20 kilómetros de distancia. La vivienda que se ofrece a este sector de población es con espacios mínimos permitidos, que como menciona Peña, cumplen con la "habitabilidad normativa" de la reglamentación establecida por los Municipios y el INFONAVIT (Peña, 2020: 101)i.

Este artículo incorpora resultados de una investigación más amplia, que fue apoyada con recursos de CONAVICONACYT. En este caso, se analizan las condiciones de habitabilidad de la vivienda construida en serie de fraccionamientos periféricos que presentan en sus casas limitantes de espacio, por su dimensión y condición física, para contribuir a la reflexión sobre las condiciones actuales del hábitat de miles de trabajadores a nivel local y nacional.

En el artículo, se utilizan los datos obtenidos para el fraccionamiento Las Haciendas que, mediante el análisis de la normatividad aplicada, es posible valorar las condiciones de la habitabilidad que ofrecen, aplicando una metodología de tipo mixta.

\section{Vivienda mínima y normatividad}

La definición de vivienda mínima tiene distintas connotaciones o interpretaciones; sin embargo, se debe entender que, si la vivienda es un bien o componente de primera necesidad, al que todo ser humano tiene derecho, su producción debe estar al alcance de todas las familias e individuos sin importar su condición social o económica (MIP-PDU, 2009). Según el artículo 4to constitucional, la vivienda debe ser digna, legal, contar con los servicios públicos básicos, y espacios habitables, que ofrezcan confort $y / 0$ bienestar, además de garantizar el acceso y el derecho a la ciudad, gozando de sus beneficios de infraestructura y equipamiento, "tales como, contar con pavimento, acceso a telefonía, servicios de luz, agua y drenaje", así como a las ventajas de contar con una casa propia (Peña y Sandoval, 2017: 28)

Esteban Molina, en "Los orígenes de la vivienda mínima en la modernidad", menciona que en el Congreso Internacional de Arquitectura Moderna (CIAM 1929-1930) se mostraron ejemplos de "vivienda para el mínimo nivel de vida", como antecedente de lo que se denominaría después vivienda mínima (Molina, 1976: 90).
En este congreso se presentaron algunas propuestas arquitectónicas, que definían la distribución y organización de una casa, donde no se tomaban en cuenta las dimensiones de los espacios, ni las medidas que requería, sino se suponían los requerimientos de la necesidad o condición del habitar (Molina, 1976: 90). Los aspectos presentados en esa época permitían diferentes interpretaciones para la elaboración de las normas que posteriormente regulan los procesos de edificación para satisfacer el "mínimo nivel de vida".

La vivienda mínima es el prototipo que, a partir del 2001, se produjo como parte de las políticas de desarrollo habitacional en el país. El costo mínimo de su producción permitió que el grupo poblacional con menos ingresos accediera a una vivienda, aunque su valor aún es cuestionable. Según la Comisión Nacional de Vivienda, la producción de la tipología de vivienda económica significó el $73 \%$ del total, pero sólo se aplicó el $34 \%$ de la inversión de los recursos INFONAVIT (CONAVI, 2013). Estos estándares mínimos han constituido la justificación del gobierno y empresas para dotar de superficies cada vez menores como patrones del modo de habitar. En el reglamento de construcción del municipio de Ciudad Juárez (Gobierno Municipal, 2004), algunas especificaciones se han reducido de tal manera que son insuficientes para garantizar las condiciones de habitabilidad recomendadas (ver figura 1).

\begin{tabular}{|c|c|c|}
\hline $\begin{array}{l}\text { Figura } 1 . \text { Disminución de } \\
\text { reglamento de } 2004 \text { y } 2015 \text { en } \\
\text { Chihuahua. }\end{array}$ & $\begin{array}{l}\text { acios } \\
\text { Munici }\end{array}$ & $\begin{array}{l}\text { los del } \\
\text { Juárez, }\end{array}$ \\
\hline REGLAMENTO DE CO & VSTRUCC & $D E L$ \\
\hline MUNICIPIO DE JUÁREZ, CHI & HUAHUA & \\
\hline $\begin{array}{l}\text { Normatividad Mínima para } \\
\text { Vivienda de Interés Social }\end{array}$ & 2004 & 2015 \\
\hline $\begin{array}{l}\text { Cajones de estacionamiento } \\
\text { por vivienda }\end{array}$ & 1 & 1.5 \\
\hline $\begin{array}{l}\text { Altura a techo desde el nivel } \\
\text { de piso terminado. }\end{array}$ & $2.40 \mathrm{~m}$ & $2.40 \mathrm{~m}$ \\
\hline $\begin{array}{l}\text { Área de al menos un cuarto } \\
\text { habitable porvivienda }\end{array}$ & $14.00 \mathrm{~m}^{2}$ & $\begin{array}{l}14.00 \\
m^{2}\end{array}$ \\
\hline $\begin{array}{l}\text { Área mínima de otros cuartos } \\
\text { habitables }\end{array}$ & $7.00 \mathrm{~m}^{2}$ & $7.00 \mathrm{~m}^{2}$ \\
\hline $\begin{array}{l}\text { El dormitorio debe contar con } \\
\text { área de guardarropa. }\end{array}$ & Si & Si \\
\hline $\begin{array}{l}\text { Dimensión mínima interior } \\
\text { entre muros paralelos de un } \\
\text { cuarto habitable }\end{array}$ & $2.50 \mathrm{~m}^{2}$ & $2.50 \mathrm{~m}^{2}$ \\
\hline
\end{tabular}

Fuente: Elaborado por Herrera para esta investigación. Las medidas asignadas son la referencia general para el diseño de la vivienda unifamiliar de interés social, que incluyen las restricciones de las reglamentaciones para lograr las autorizaciones de los trámites y permisos de construcción. El reglamento de construcción establece 
que la iluminación y ventilación natural de los cuartos habitables [recámara, estancia 0 sala] son fundamentales para la higiene, con el cambio de aire en el interior de las viviendas; en este caso, todas las habitaciones habitables deben contar con una ventana al exterior. El tamaño de la ventana es el 10 por ciento de la superficie del espacio del área del piso y en ningún caso inferior a un metro cuadrado (Gobierno Municipal, 2004). En los espacios como el baño y la lavandería [no habitables] la ventana no debe ser menor al 20 por ciento de la superficie del piso y en ningún caso más pequeña de 0.16 metros cuadrados, es decir una ventana de 0.40 $\mathrm{m} \times 0.40 \mathrm{~m}$ (Gobierno Municipal, 2004).

Los espacios habitables y no habitables están definidos por la función que cumplen para las actividades esenciales de un hogar; es decir, dormir, alimentarse, cocinar y estar, y que corresponden a la sala, cuarto de estudio, biblioteca, comedor, cocina y dormitorios. Los no habitables serían de servicio como el baño, lavandería, cuarto de climatización, closet y circulaciones (Gobierno Municipal, 2004).

El reglamento de la ciudad también establece que toda vivienda debe considerar al menos dos espacios habitables, uno puede ser la cocina; de los no habitables al menos un baño que incluya lavabo, inodoro y regadera (Gobierno Municipal, 2004).

En cuanto a la exigencia del reglamento, de que toda vivienda debe localizar el estacionamiento dentro de la propiedad, hace referencia al interés de una ciudad supeditada al uso del automóvil, aspecto que no corresponde con el sector de población al cual se oferta la propiedad y que difícilmente puede acceder a un vehículo. Este tipo de vivienda plantea los siguientes espacios:

\begin{tabular}{|c|c|c|c|}
\hline \multicolumn{4}{|c|}{$\begin{array}{l}\text { Figura 2. Cuadro de espacios mínimos según el reglamento } \\
\text { de construcción. }\end{array}$} \\
\hline CANT. & $\begin{array}{l}\text { TIPO DE } \\
\text { ESPACIO }\end{array}$ & ESPACIO & $\begin{array}{c}\text { MINIMO } \\
\text { ACEPTABLE }\end{array}$ \\
\hline 1 & $\begin{array}{l}\text { Espacio } \\
\text { habitable }\end{array}$ & $\begin{array}{l}\text { Sala, comedor } \\
\text { y/o recámara }\end{array}$ & $14 \mathrm{~m}^{2}$ \\
\hline 1 & $\begin{array}{l}\text { Espacio } \\
\text { habitable }\end{array}$ & Cocina & $7 \mathrm{~m}^{2}$ \\
\hline 1 & $\begin{array}{l}\text { Espacio no } \\
\text { habitable }\end{array}$ & baño & $3 \mathrm{~m}^{2}$ \\
\hline \multicolumn{3}{|c|}{$\begin{array}{l}\text { VIVIENDA MINIMA CON DOS } \\
\text { ESPACIOS HABITABLES }\end{array}$} & $38 \mathrm{~m}^{2}$ \\
\hline
\end{tabular}

Fuente: Elaboración propia.

En el análisis de la figura 2, el concepto de vivienda digna y mínima, según el reglamento de construcción, especifica cierto número de metros cuadrados, para con ello garantizar la habitabilidad, y se obtiene el permiso para el desarrollo habitacional o la edificación (Gobierno Municipal, 2004).
Los estándares de la vivienda mínima o pie de casa habitación, determinados en la normatividad municipal, no corresponden a la trascendencia de la función social que tiene para la mejora de la calidad de vida de una familia, incluso incumple con la condición de espacio digno y habitable según se expone en el Artícu lo 4to de la Constitución.

Los espacios de construido mínimo, cuya localización es periférica, limita a los habitantes para acceder a los beneficios de equipamiento y servicios que ofrece la ciudad, deberían ser compensados con los denominados "regalos locacionales", que deben ofrecerse y subsanar los gastos adicionales del salario que se utiliza para el desplazamiento respecto a otros sectores más cercanos al trabajo (Wingo, 1961 en Abramo, 2010). Estos beneficios podrían ser: un terreno más amplio, mejores áreas verdes, servicio de movilidad pública y eficiente, actividades culturales, atención a la salud a bajo costo, entre otros.

El Plan de Desarrollo Urbano de Ciudad Juárez menciona que el tamaño mínimo del predio es de 120 metros cuadrados para la vivienda unifamiliar y con un frente no menor a 6 metros. En vivienda en con dominio, el terreno menor es de 180 metros cuadrados (IMIPPDU, 2009).

El Instituto del Fondo Nacional de la Vivienda para los Trabajadores (INFONAVIT), establece las dimensiones mínimas que deben tener el predio y la vivienda. En el caso del estado de Chihuahua estas dimensiones son las siguientes:

\begin{tabular}{|c|c|}
\hline $\begin{array}{c}\text { Figura 3. Espacios mínimos según la cédula publicada por } \\
\text { la Subdirección General Técnica y de Delegaciones } \\
\text { Gerenciales de Valuación Inmobiliaria del INFONAVIT. }\end{array}$ \\
\hline ESPACIO & SUPERFICIE \\
\hline Lote mínimo & $120 \mathrm{~m}^{2}$ \\
\hline Frente no menor & $7 \mathrm{~m}$ \\
\hline Cocina no menor & $3 \mathrm{~m}^{2}$ \\
\hline Baño no menor & $1.5 \mathrm{~m}^{2}$ \\
\hline Recámara & $6 \mathrm{~m}^{2}$ \\
\hline Estancia o sala & $13 \mathrm{~m}^{2}$ \\
\hline $\begin{array}{c}\text { VIVIENDA MINIMA CON DOS ESPCIOS } \\
\text { HABITABLES }\end{array}$ & $23.5 \mathrm{~m}^{2}$ \\
\hline
\end{tabular}

Fuente: Elaboración propia

La cédula que publicó la Subdirección General Técnica y de Delegaciones Gerenciales de Valuación Inmobiliaria, tiene como propósito identificar los requerimientos básicos de la vivienda, establecidos por la autoridad oficial a nivel estatal y/o municipal. La información, al ser publicada por parte de una dependencia como INFONAVIT, lleva a respetar esa reglamentación como el marco legal que favorece a las constructoras, ya que se disminuye aún más el área construida por espacio habitable de $14 \mathrm{~m}^{2}$ a $6 \mathrm{~m}^{2}$ y para el no habitable, de $3 \mathrm{~m}^{2}$ 
a $1.5 \mathrm{~m}^{2}$. El impacto en la calidad habitacional, en cu anto al tamaño de una vivienda con dos espacios habitables, cocina y baño, decrece de $38 \mathrm{~m}^{2}$ a $23.5 \mathrm{~m}^{2}$, que se otorgan a la mayoría de los créditos de vivienda para los trabajadores con salario establecido (ver figura 3).

\section{Habitabilidad y calidad de vida en la vivienda}

La habitabilidad, hace referencia al término de calidad en el espacio que se habita, y remite a la calidad de vida (Moreno, 2008), término que desde la década de los setenta se define como el conjunto de condiciones que generan bienestar y que contribuyen al logro de la potencialización de la vida social de los individuos; se aplican distintos métodos para medirla, ya que no existe una única teoría que defina y explique el modo de habitar. Es un concepto que considera la construcción compleja y multifactorial, existiendo una gran cantidad de indicadores que permiten medirla con el análisis objetivo y perceptual. En cuanto a la habitabilidad, se plantean algunas características que "involucran el acceso a un hábitat justo, [...] la existencia de una vivienda de calidad, seguridad en la tenencia, la cercanía a escuelas, transporte público y hospitales" (Páramo et al., 2018: 347).

La habitabilidad identifica aspectos cualitativos y cuantitativos que valoran las experiencias subjetivas y objetivas de los individuos, como "la cultura y el lenguaje de los ocupantes" (Peña y Sandoval, 2017: 28), y que Macías y De Hoyos definen como "la estética de la habitabilidad", es decir que se "manifiesta de modo distinto, el sentido de apropiación, ocu pación, identidad y significación del espacio -modos de habitar-" (Macías y De Hoyos, 2015: 274).

La habitabilidad, se puede comprender en escenarios diferentes, con indicadores específicos que permitan estimar y determinar satisfactores que requieren las personas en distintos espacios. "Es posible pensar en la relación cosmogónica con el espacio habitado, pues la forma de habitar no sólo implica ocupar un lugar construido físicamente, sino mirar la manera de ocuparlo" Macías y De Hoyos, 2015: 274).

Por su parte, Moreno (2008) menciona que la habitabilidad se debe medir a partir de lo externo o el entorno y lo interno o espacio habitable, de la vivienda. Por otro lado, Rueda (1999) también plantea que el bienestar debe ser analizado a partir de lo externo y lo interno, donde lo interno se refiere al espacio y lo perceptual, mientras que lo externo aborda lo físico y social del contexto.

Las distintas acepciones planteadas, obligan a considerar la habitabilidad ligada al espacio y características del medio ambiente y el entorno social, desde una postura subjetiva y objetiva. Ambos componentes plantean que de manera individual y colectiva se logre la satisfacción de ocupar y habitar un espacio, en un lugar o sector determinado; "no podemos pensar en construir viviendas justas si no hay barrios justos. Resulta imperioso contar con una mirada integral para que exista un acceso justo al hábitat" (Bernat, 2020: 732).

\section{Metodología}

En este trabajo se aporta información parcial de otra investigación más amplia, realizada con recursos de CONAVI-CONACYT. La metodología aplicada es de tipo mixta, debido a que se cuenta con datos del método cuantitativo (gráficos, matrices, dibujos) y del método cualitativo (la opinión de los habitantes). La investigación se realizó en tres ciudades de México: Mexicali, Baja California; Mérida, Yucatán y Ciudad Juárez, Chihuahua. El objetivo considerado fue: analizar indicadores de habitabilidad ambiental para fraccionamientos con vivienda construida en serie. Se observaron diferentes fraccionamientos en las tres ciudades; para este caso, se utilizan parte de los resultados obtenidos sobre el fraccionamiento Las Haciendas en Ciudad Juárez, Chihuahua. Para dar respuesta al objetivo se realizó lo siguiente:

1) Revisión de más de 900 autores que atienden el concepto habitabilidad, para elaborar un eje teórico con los conceptos que definen las principales ideas de la investigación.

2) Selección de la zona de estudio, mediante la caracterización del tipo de vivienda, localización en la ciudad, tipo de población, ubicación conforme a las zonas industriales y la seguridad en el sector, que favorecieran la aplicación de instrumentos de campo.

3) Elaboración de cuestionarios piloto sobre habitabilidad ambiental, urbana y micronegocios. Se definió un modelo final que facilitara el trabajo de campo y posteriormente, el análisis. La muestra general se calculó al considerar el total de viviendas habitadas de tres fraccionamientos que abarcan la zona de estudio, con un universo de 5454 casas, según el censo de población y vivienda del 2010 y del caso de estudio de 4241 viviendas, de las cuales, 1,400 estaban desocupadas (INEGI, 2010).

4) El cálculo de la muestra planteó un nivel de confianza del 95 por ciento, margen de error del 5 por ciento y la probabilidad de ocurrencia del 30 por ciento. El resultado para los tres fraccionamientos en Ciudad Juárez fue de 219 encuestas. La muestra para el área de estudio, fraccionamiento "Las Haciendas" (el de mayor extensión), fue de 114 cuestionarios.

La aplicación de la encuesta fue por estratificación proporcional y al azar. Es decir, se hizo una distribución equivalente entre las 87 manzanas y el total para aplicar de 114 cuestionarios. Se distribuyó de forma aleatoria, 
equitativa y representativa en el área de estudio. Se aplicó a personas mayores de 18 años que radicaban en la vivienda.

5) El trabajo de campo se hizo con el acopio y obten ción de información directa, cuidando la calidad de la misma, mediante mecanismos de control del levantamiento y revisión de llenados.

6) Para la obtención de resultados y el análisis, se procedió a la sistematización de datos en software especializado SPSS-19 y para las conclusiones, se recurrió a la triangulación de la información, identificando los hallazgos más relevantes de tipo objetivo y subjetivo.

\section{Resultados}

El fraccionamiento "Las Haciendas, está localizado al sur oriente de la ciudad, dentro de la estructura urbana y organización espacial con mayor crecimiento u rbano en las dos últimas décadas (ver figura 4).

Figura 4. Traza urbana de Ciudad Juárez (2016) y ubicación del área de estudio "Las Haciendas".

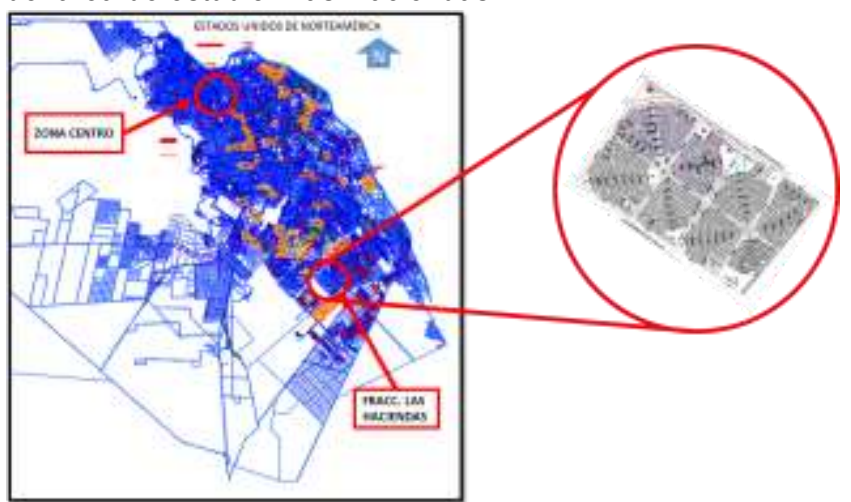

Fuente: Elaboración propia.

"Las Haciendas", con relación a otros fraccionamientos, son:

a) Traza radial, poco común en la ciudad.

b) Ubicación a 500 metros de los parques industriales (cercano a la planta maquiladora más grande de la ciudad-Electrolux).

c) Abandono de vivienda con el 33 por ciento.

d) Aplicación de las encuestas según la ponderación realizada de acuerdo cantidad de viviendas entre manzanas.

El fraccionamiento cuenta con 4241 viviendas, en una superficie de 1'066,670.90 metros cuadrados, registra 1400 casas desocupadas, según el Instituto Nacional de Geografía y Estadística (INEGl, 2010) y una población de 8950 habitantes. La ocupación es de 3.15 personas por casa. La ubicación del fraccionamiento, con respecto al centro de la ciudad, es de 30 kilómetros aproximadamente (ver figura 4).
Figura 5. Panorámica del fraccionamiento Las Haciendas.

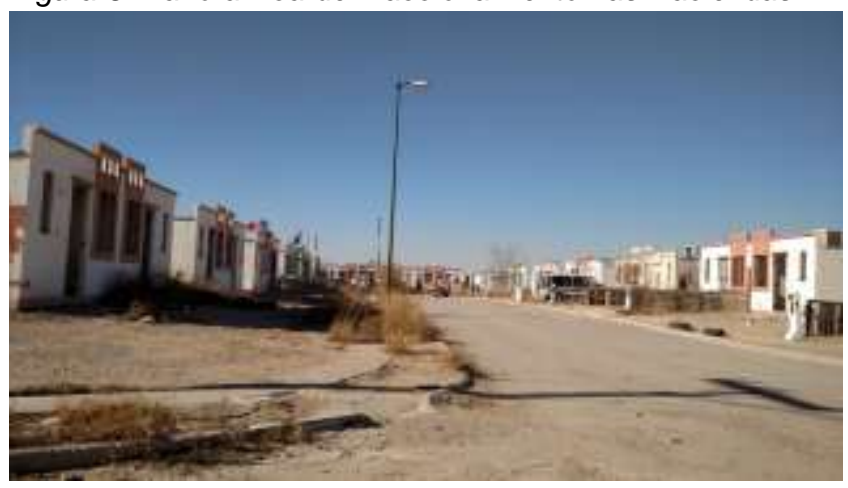

Fuente: Autor, 2019.

La imagen urbana del sector es precaria, debido a la concentración de casas abandonadas, calles y banquetas en deterioro, acelerada condición de obsolescencia, que en menos de 5 años se visualiza un entorno con mayor deterioro y obsolescencia, con un proceso de consolidación (ver figura 5).

Los ambientes interiores de las viviendas dan muestra de condiciones espaciales reducidas con las siguientes tipologías:

Casa tipo 1: con 40.20 metros cuadrados construidos, incluye dos recámaras, un espacio múltiple para sala y/o comedor y cocina con espacio insuficiente para la estufa, el refrigerador y fregadero, con instalaciones hidráulicas, sanitarias y de gas fijas (ver figura 6).

Figura 6. Planta arquitectónica de las 2 Tipologías de vivienda en el área de estudio.
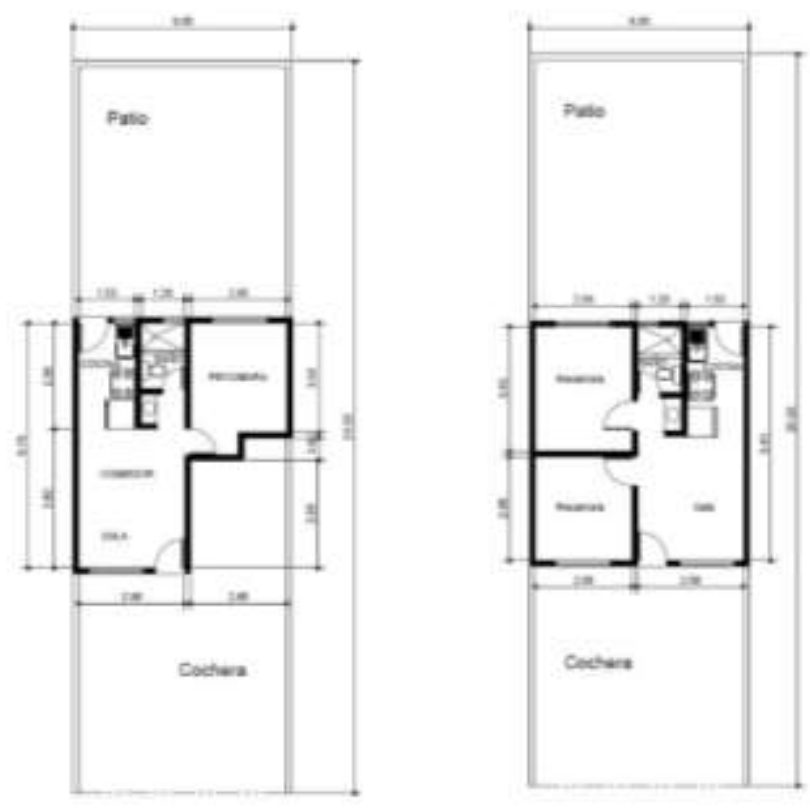

Fuente: Elaboración propia.

Casa tipo 2: es de menor tamaño, con 32.52 metros cuadrados de construcción, incluye una recámara, área 
de cocina similar a la anterior (espacio limitado), el espacio múltiple para sala y/ o comedor. Este prototipo, al igual que el primero, no cumple con las dimensiones mínimas de un espacio habitable (tener $2.50 \mathrm{~m}$. libres entre mu ros paralelos) (ver figura 6).

En cuanto a la disposición para espacios de guardado, en ambas tipologías, no se proveen, falta área de closet o ropero, por tanto, se incumple según el reglamento (ver figura 6).

Al ingresar a una vivienda abandonada, se obtuvo una imagen del interior (ver figura 7). En esta figura se ofrece una idea del espacio de la cocina con puerta de salida al patio posterior y los accesos al baño y recámara, que dan cuenta del espacio habitable construido. El ambiente es austero, sin colores y con acabados mínimos (al interior yeso y bloque con pintura al exterior). El espacio reducido y falta de amplitud dificulta el ingreso de muebles voluminosos y que en general, se obstruyen las circulaciones. Las familias no han podido invertir mejoras en la vivienda, debido al adeudo desde que la adquirieron, mostrando condiciones de deterioro. El espacio de la sala y/o comedor, corresponde al sitio desde donde se tomó la fotografía.

Figura 7. Vista interior de una vivienda abandonada.

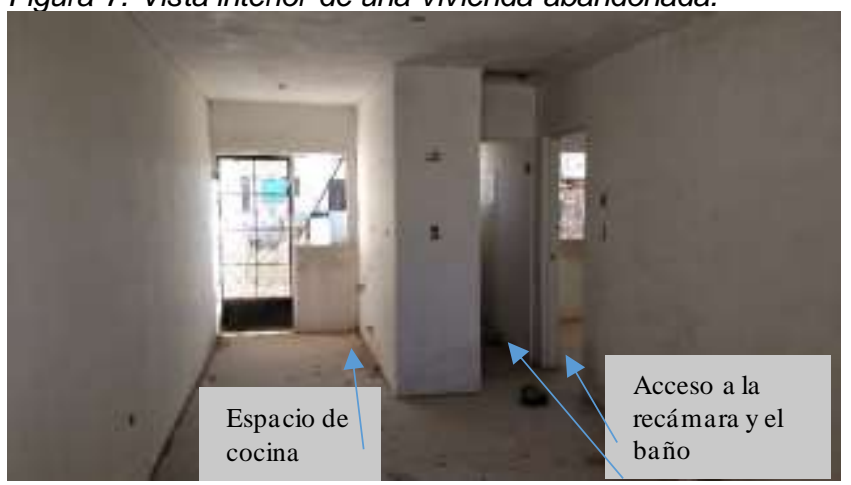

Fuente: Peña, 2019.

El incumplimiento de la normatividad y el producto de vivienda que se oferta en la ciudad, se otorgan como una opción inevitable para los beneficiarios, teniendo registros de que la misma vivienda se ha abandonado más de 10 veces en menos de 12 años, resultado de factores de localización, costos de traslado y falta de seguridad (López, 2017)ii. En el análisis de resultados se podrá conocer la opinión o percepción de los usuarios.

\section{Análisis de resultados}

Se elaboraron tres tipos de cuestionarios para conocer la percepción que tienen los ocupantes sobre la habitabilidad ambiental de la vivienda, la urbana y micronegocios del contexto inmediato que habitan. En el tema sobre habitabilidad ambiental de la vivienda se opinó sobre la funcionalidad, dimensionamiento, significado y valoración de la vivienda. Respecto a la habitabilidad urbana, se comentó sobre seguridad, patrullaje, espacios verdes y calidad del espacio público y servicios, entre algunos ${ }^{\mathrm{iii}}$.

Las familias identifican dificultades en el uso de la vivienda, debido a que la movilidad interior es ineficiente para que las actividades cotidianas: al cocinar, estar en el espacio de estancia o en las recámaras y baño, ya que el mobiliario que tienen no corresponde a las dimensiones mínimas de la construcción. En opinión de los usuarios, 39 por ciento evaluó mal el funcionamiento dentro de la casa, 24 por ciento regular y 37 por ciento bien. Limitantes que en términos de la psicología ambiental generan frustración y conflictos entre los ocupantes. Incluso se desarrolla un tipo de "impotencia aprendida" que tiene repercusiones en la falta de colaboración y compromiso colectivo a lo largo de la vida (Cohen, 1980: 95)iv. Es pertinente mencionar que para muchos de los habitantes es la primera y quizá la única opción de contar con una vivienda propia.

En cuanto a la dimensión de las viviendas, es relevante entender que los prototipos se ofertan por el costo de los metros cuadrados construidos, esto significa que el financiamiento no se define por el perfil de las familias. Según otros estudios realizados por los autores, las familias eligen, en muchas ocasiones, sin visitar o conocer la propiedad, aspecto que Peña define como elegibilidad y que se determina por las oportunidades que tuvieron las familias para decidir mediante el conocimiento informado, logrando que sea un acto consciente y no una decisión bajo ignorancia (2020: $101)^{\mathrm{v}}$.

El dimensionamiento de la vivienda, fue evaluado por 33 por ciento como buena, 27 por ciento regular y el 40 por ciento como mala. Las dimensiones son parte del resultado de la aplicación del mínimo establecido en la normatividad y reglamentos de construcción para la vivienda en serie. La habitabilidad está condicionada por la cantidad de ocupantes y el uso permanente de los espacios. Se observó que muchas de las viviendas no cuentan con parte del mobiliario, ya sea para mejorar la movilidad o porque no se consigue con las dimensiones requeridas para ese tamaño.

Los habitantes plantean que la funcionalidad $y$ dimensiones de la vivienda se dificultan para 38.6 por ciento, al evaluarla como mala, para 21.9 por ciento como regular y para 39.5 por ciento como buena. En cuanto al dimensionamiento, 38.1 por ciento consideran que es pésimo y malo; 28.4 por ciento menciona que es regular y 33.5 por ciento lo definen como bueno y excelente. Se observa que las familias integradas por 40 más personas lo califican entre pésimo, malo y regular, que corresponde al 52.8 por ciento de los encuestados. La falta de espacio se comprende debido a que el número de ocupantes interfiere en la habitabilidad, por 
ejemplo, en la muestra obtenida, 5 por ciento es habitada por una o dos personas; 52 por ciento por tres y cuatro personas; 36 por ciento por cinco o seis personas y, el siete por ciento restante, se ocupa por siete a once personas. La normatividad plantea que cada espacio habitable puede ser ocu pado por 2 personas y hasta 2.5 personas, según la CONEVAL (2020). Es decir que cualquier vivienda con más de 4 ocupantes presenta algún grado de hacinamiento.

La dinámica de empleo en la ciudad puede ejemplificar un uso con menor hacinamiento en la vivienda; sin embargo, en esta situación del resguardo por la pandemia por Covid-19, la presencia permanente de personas ha intensificado las condiciones de estrés en sus ocupantes; hay un incremento del 81 por ciento para llamadas de apoyo en las instancias de la Secretaria de Salud en el área de atención mental.

La coyuntura actual de la pandemia por Covid-19, don de miles de familias se resguardan en casa y pretenden evitar el contagio, se complica, ya que la sana convivencia en un ambiente tan limitado es un detonante de estrés ambiental, debido a la aglomeración, falta de comunicación por ruido, incremento del desorden y falta de higiene; pérdida de privacidad que propicia violencia, $y$, de acuerdo con los registros elaborados, se presenta: depresión (73.7\%), tristeza (34.8\%) y ansiedad (29.2\%) en un grupo de población distinto, pero que habitan en estos fraccionamientos ${ }^{\text {vi }}$. Esto nos lleva a reflexionar que la actividad cotidiana ha sido irrumpida con la permanencia de la familia en casa, que durante dos meses no fueron al trabajo, se realizaron actividades escolares con los hijos y se tenían que resolver las exigencias de atención del hogar.

Al respecto, las actividades laborales fueron clausuradas por dos meses, debido al rápido contagio que se dio en la ciudad, aunado que la industria manufacturera es no esencial (electrónica y automotriz). Posteriormente, se tuvo un retorno parcial de tres días por semana hasta agosto y apenas el último mes, regresó todo el personal. En ese sentido, las familias resguardadas en casas mínimas, con patios reducidos, para lograr la solicitada sana distancia, se ve afectada, si alguno de los residentes ha logrado contagiarse de la Covid-19, y tratando de mantenerse aislado, haría uso exclusivo de la única recámara y baño, mantendría a todos los otros integrantes en un menor espacio, incrementando los problemas de estrés diurno y nocturno.

A nivel local, 65.26 por ciento trabaja en el sector secundario de la industria maquiladora y 31.12 por ciento en el sector terciario (INEGI-ENOE, 2019). Esto podría suponer que existen horarios escalonados, en donde los ocupantes no coinciden en el hogar. Esto podría ser posible, aunque la dinámica económica se concentra en turnos matutinos y vespertinos, y con menos afluencia en el nocturno. Los obreros de maquiladoras cumplen horarios de hasta 12 horas diarias, cuando existe excedente de trabajo. Esto implica que los horarios no se establecen por el trabajador sino por la empresa, que puede modificarlos según convenga a la producción, aspecto que complejiza más la actividad cotidiana en el hogar, ya que mientras unos duermen, otros hacen actividades escolares en casa.

En cuanto a la valoración que tienen de la vivienda los residentes, al contar con una vivienda propia y lugar que disminuye la vulnerabilidad de carecer de casa, sin importar cuan alejada se ubique, siendo asequible a su nivel de ingresos, refleja la opinión más positiva respecto al nivel de satisfacción, como se muestra en la figura 8.

Figura 8. Opinión sobre la satisfacción con la vivienda.

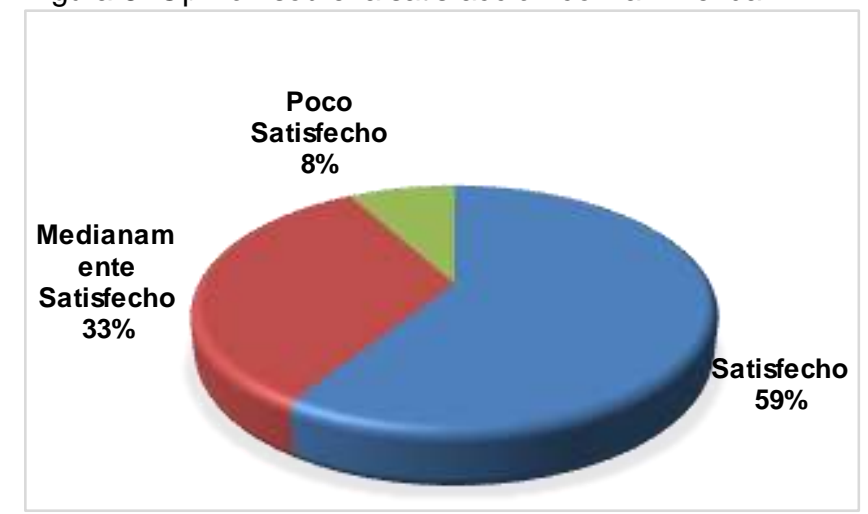

Fuente: Elaboración propia.

La opinión de satisfacción con la vivienda que tienen, refleja que 59 por ciento está satisfecho, 33 por ciento medianamente satisfecho y 8 por ciento poco satisfecho. Esta valoración se asocia con que han resuelto una necesidad apremiante de contar con un lugar propio donde vivir, disponer de una propiedad que se con vierte en el patrimonio familiar y en algunos casos, es una inversión que les da seguridad y estabilidad emocional. Un efecto que ha influido en el abandono de vivienda, es el incremento de gastos, debido a la ubicación de ésta, respecto de la ciudad. En ese sentido, se planteó la posibilidad para elegir cambiarse con el mismo financiamiento, para que el encuestado se posicionara respecto a esta posibilidad, por lo que, 46 por ciento manifestó que no, pero 47 por ciento mostró interés en hacerlo y, 7 por ciento expresó que tal vez. Las razones expresadas para realizar el cambio, se vieron motivadas por el interés de habitar más cerca de la familia, en un sitio más céntrico o que cuente con otros servicios o propiedades más amplias, como se muestra en el gráfico de la figura 9. 
Figura 9. Opinión sobre cambiarse de vivienda.

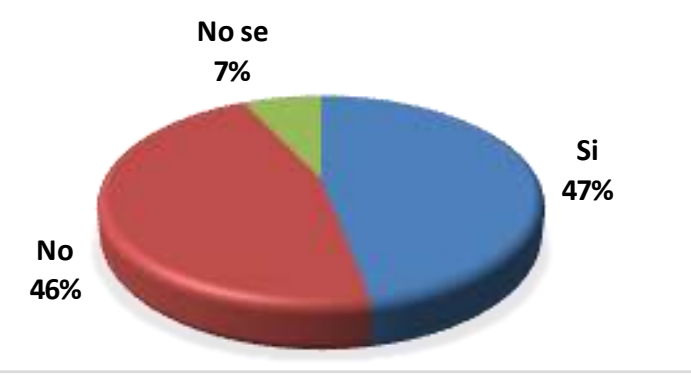

Fuente: Elaboración propia.

En cuanto a las opiniones respecto al espacio urbano, se plantean las relacionadas con la seguridad, áreas verdes, calidad de calles y banquetas, así como la atención del servicio de patrullaje.

La opinión que tienen sobre la seguridad refleja que existen actos delictivos que vulneran la habitabilidad urbana, robos a vivienda y a las personas en la vía pública. En este sentido, 33 por ciento considera muy inseguro e inseguro el habitar en esos lugares; 34 por ciento regular y 33 por ciento como seguro y muy seguro. (Ver figura 10). Esta percepción se relaciona con la frecuencia en el servicio de patrullaje, pues 65 por ciento, que es de dos a tres veces por semana, 17 por ciento semanal y 7 y 11 por ciento dice que nunca y casi nunca, respectivamente.

Figura 10. Opinión sobre el ambiente de seguridad.

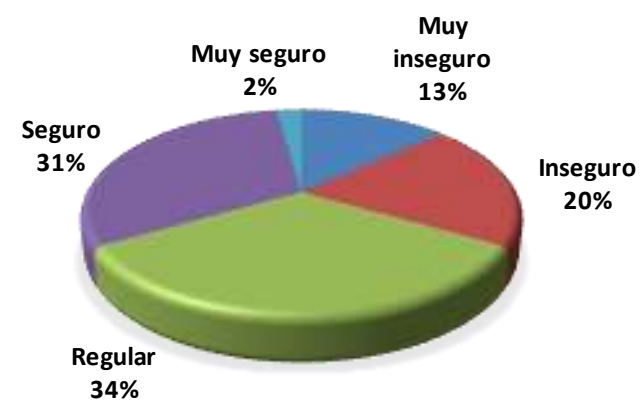

Fuente: Elaboración propia.

El deterioro del espacio urbano vigente (ver figura 5), refleja el nivel de abandono que prevalece en las viviendas, debido a las deficiencias en las soluciones urbanas que no favorecen la convivencia, la identidad de barrio o el uso del espacio común. En el fraccionamiento estudiado, la estructura urbana cuenta con pocas vialidades principales y múltiples calles terciarias sinuosas, poco funcionales, y que favorecen el abandono y falta de identidad, que reflejan la ciudad supeditada al uso del automóvil.

La organización espacial del área verde tiene una localización deficiente que inhibe la apropiación y muestra la falta de conservación y de uso por parte de los residentes, ya que se aprecian ambientes en deterioro y con imagen urbana precaria. Esta conservación no es asumida por los habitantes, debido a la inexistente experiencia de colaboración y participación comunitaria, pues la actividad asalariada "determina que las familias tengan que destinar más tiempo del trabajo total de sus miembros a la obtención de ingresos, en detrimento de los trabajos de cuidado" (Pérez, 2000:

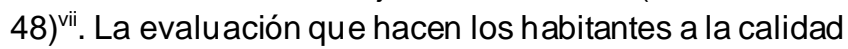
de espacios verdes es: 83 por ciento la considera mala, 13 por ciento regular y 4 por ciento buena. Estas opiniones corresponden a la realidad de abandono y rápido deterioro que impacta el patrimonio y que en muchas ocasiones, se sigue pagando, aunque se haya abandonado.

El ambiente de deterioro que prevalece en calles y banquetas también es valorado por la mayoría (73\%) por sus malas condiciones, pocos (20\%) lo perciben como regular y solo 7 por ciento como bueno.

La calidad precaria de la vivienda, el deterioro y obsolescencia del espacio urbano, es reflejo de la aplicación de una normatividad que regula lo mínimo, que es laxa y carece de control o seguimiento posterior a la ocupación, ya que al terminar de vender las casas, los fraccionadores carecen interés en su conservación, y en gran parte de las ocasiones, el municipio no se ocupa de ello. Esto da un efecto o sensación de un paisaje desolador, de abandono que ni autoridad ni empresa y mucho menos los habitantes, procuran mejorar.

En términos de habitabilidad, tanto la percepción social como la apariencia física del espacio son resultado de la concepción de vivienda como un bien de mercado y no como un bien social, indicador de progreso y calidad de vida.

\section{Conclusiones}

La calidad de los bienes de una familia se centra en la visión legal y social que se desarrolla en una comunidad, como resultado de la normatividad y aplicación de planes y reglamentos que definen el límite del progreso. Si basamos estas normas para cumplir con lo mínimo, sin mediar con ello las profundas carencias que conlleva, es poco factible enderezar el rumbo de la política habitacional. Por consiguiente, se deben revisar, actualizar y direccionar los reglamentos para resolver las necesidades de las familias, no solo la oportunidad de negocio, sino el bienestar social.

Entender que la vivienda mínima es un bien social que debe ser diseñada para atender las necesidades de habitabilidad, implica tener presente las condiciones de un contexto urbano para la socialización y para hacer ciudadanía, al ponderar otros parámetros de espacio y de acceso a la misma, que no privilegie únicamente el factor económico. 
La espacialidad y altura interior de áreas habitables, se plantea con el mínimo y en muchos casos se incumple. Lograr mejores opciones de espacialidad interior en las viviendas debe procurarse como política habitacional, que mediante estímulos (tipo fiscal) pueda revertir las deficiencias observadas para el desarrollo humano.

La introducción de generación de energía, de cuidado del agua, de espacios flexibles que sean subdivididos al gusto de las familias, y de otras alternativas de financiamiento (autoproducción, familiar, de uso mixto, etc.) y con involucramiento y participación de los habitantes, se puedan detonar acciones de emprendimiento, rehabilitación y mejora de ambientes.

Finalmente, la aplicación de la normatividad debería premiar la innovación e impulso de las condiciones de progreso de un lugar, validando con ello, el derecho a una vivienda digna y decorosa; desalentando acciones que incrementen la precarización del patrimonio habitacional.

\section{Referencias}

Abramo, Pedro (2010). Mercado y orden urbano. Del caos a la teoría de la localización residencial. Traducción: Melba Rubiano. Universidad Externado de Colombia.

Bernat, María Sofía. Modos de habitary políticas públicas: reloca liza ción de un asentamiento en La Plata, Argentina. Estudios Demográficos y Urbanos, vol. 35, núm. 3 (105), pp. 727-759.2020

Cohen, S. (1980). Aftereffects of stress on human performance and social behavior: A review of research and theory. Psychological Bulletin. No. 88, pp. 82-108. USA: American Psychological Association. https://www.researchgate.net/publication/15796493 Aftereffects of st ress on human performance and social behavior A review of rese arch and theory

CONAVI, Comisión Nacional de Vivienda. (2020). Programa de Vivienda Social. Disponible en línea: https://www.gob.mx/conavi/acciones-yprogramas/s177-programa-de-vivienda-social-pvs

CONEVAL (2018). Estudio diagnóstico del derecho a la vivienda digna y decorosa 2018. México: Comisión Nacional de Evaluación de la Política de Desarrollo Social. Disponible en línea: https://www.coneval.org.mx/Evaluacion/IEPSM/Documents/Derechos Sociales/Estudio_Diag_Vivienda_2018.pdf

IMIP/PDU (2009). Plan de Desarrollo Urbano. Gobierno Municipal e Instituto Municipal de Investigación y Planeación, Ciudad Juárez, México.

INEGI (2010). Censo de población y vivienda 2010, Instituto Nacional de Esta dística, Geografía e Información. México.

INEGI (2019). Encuesta Nacional de Ocupación y Empleo (ENOE). Primer trimestre de 2019. Principales indicadores laborales de las ciudades. Mayo de 2019. México: Instituto Nacional de Estadística, Geografía e Informática

López Nava, V. M. (2017). Problemática de la vivienda en ciudad Juárez. Tesis doctoralinédita, México: Colegio de Chihuahua.

Macías, Yatzin Yuriet; y De Hoyos, Jesús Enrique (2015). El diseño del espacio público en el contexto de la habitabilidad del colectivo social. En "Diseño para la calidad de vida del espacio habitable". (pp. 271298). Toluca, México: Universidad Autónoma delEstado de México.

Molina Ramírez, Esteban., (2014). Orígenes de la vivienda mínima en la modernidad, Tesis de Maestría, Universidad Nacional de Colombia, https://www.academia.edu/35799524/Or\%C3\%ADgenes_de la vivien da_m\%C3\%ADnima en la modernidad
Moreno Olmos, Silvia Haydeé (2008). La habitabilidad urbana como condición de calidad de vida. Pa lapa, vol. III, núm. II, julio-diciembre, 2008,pp. 47-54, Universidad de Colima, México.

Páramo, Pablo; Burbano, Andrea; Jiménez-Domínguez, Bernardo; Barrios, Verónica; Pasquali, Carlota; Vivas, Fabiola; Moros, Oscar; Alzate, Mónica; Jaramillo-Fayad, Juan Carlos \& Moyano, Emilio (2018). La habitabilidad del espacio público en las ciudades de américa latina. Avances en Psicología Latinoamericana, 36 (2), pp. 345-362. http://www.scielo.org.co/pdf/apl/v36n2/1794-4724-apl-36-02$\underline{00345 . p d f}$

Peña, Leticia \& Sandoval, Lidia (2017). Ciudad Juárez: deterioro y abandono de vivienda. Revista Ciudades 113, enero-marzo, 2017 (pp. 28-36). México: Red Nacionalde Investigaciones Urbanas (RNIU)

Peña Barrera, Leticia (2020). Microscopía urbana. Una visión de la reurbanización de barrios. México: Universidad Autónoma de Ciudad Juárez.

https://www.researchgate.net/publication/342851087 MICROSCOPIA URBANA Una_vision de la reurbanizacion_de barrios

Pérez Molina, Ivet Miriam (2005). Capítulo 2. Relaciones de convivencia familiares. En Incide Social, A.C. Investigación para la elaboración de un Plan de Acción Social Concertado en Ciudad Juárez. México: Consejo Ciudadano de Desarrollo Social. (pp. 24-45. https://incidesocial.org/wp-content/uploads/PLAN-DEACCI\%C3\%93N-SOCIAL-CONCERTADO-EN-CD.JU\%C3\%81REZ-1.pdf

DOECH (2004). Reglamento de construcción Municipio de Ciudad Juárez, Publicado en el Periódico Oficial del Estado de Chihuahua. 09 de octubre del 2004, México.

Rueda, Salvador (1999). Modelos e indicadores para ciudades más sostenibles. Taller sobre indicadores de huella y calidad ambiental urbana. Departamento de Medio Ambiento de la Generalitat de Catalunya.

Ruvalcaba, Rosa María \& Schteingart, Martha (2012). Ciudades divididas, desigualdad y segregación social en México. El Colegio de México.

Wingo, Lowden (1961). Transportation and urban land. USA: Resources for the future. En Abramo, Pedro (2010). Mercado y orden urbano. Del caos a la teoría de la localización residencial. Traducción: Melba Rubiano. Universidad Externa do de Colombia.

NOTAS

\footnotetext{
i La habitabilidad normativa "corresponde a los requerimientos establecidos en reglamentos de construcción y que pueden cumplirse en todo proyecto o urbanización".

ii En esta investigación López Nava, de 49 casos analizados de nuevos fraccionamientos, la vivienda que más ocasiones se revendió fue 10 veces y de 3 sucesiones la que menos. Este modelo produce beneficio a las empresas que revenden.

iii Información directa obtenida con encuestas y mediciones aplicadas en el sector de estudio. El instrumento fue elaborado para la investigación CONAVI-CONACYT de 2016 a 2018.

iv Cohen (1980: 95) plantea que "la exposición a un estrés impredecible o incontrolable va seguida de una menor sensibilidad para los demás. [...] una disminución en la ayuda, una disminución en el reconocimiento de la s diferencias individuales y un aumento en la agresión".

${ }^{v}$ La autora identifica que las empresas no buscan soluciones innovadora s sino únicamente cumplir con los mínimos que establece la normatividad, plantea que la "habitabilidad superior: establece nuevos valores o referentes de calidad residencial que deben perdurar en el largo plazo, garantizando la calidad de estos en el tiempo" (Peña, 2020: 101)

vi Resultados de una investigación en curso y en proceso de publicación, elabora da por Peña en 2020.

vii Según Pérez Molina (2000: 48), "la inserción de las mujeres en las actividades de la maquila, si bien brinda oportunidad para que ellas trabajen, tienen efectos negativos en su vida y en la de sus familias que contribuyen al malestar social".
} 